\title{
Le modèle explicatif des maladies infectieuses associées à la misère et à la pauvreté : l'expérience espagnole et italienne dans la première moitié $\mathrm{du} \mathrm{XX}^{\mathrm{e}}$ siècle
}

\author{
Lucia Pozzi, Josep Bernabeu-Mestre et María Eugenia Galiana-Sánchez
}

\begin{abstract}
Résumé
Le travail aborde l'étiologie sociale des maladies infectieuses associées à la pauvreté, maladies dominantes en Espagne et en Italie dans la première moitié du $\mathrm{XX}^{\mathrm{e}}$ siècle. La première partie analyse le cadre explicatif de l'interaction entre agents infectieux, individus vulnérables ou susceptibles d'être malades et environnements morbides qui facilitent la contagion par l'action des fomites et des vecteurs passifs des maladies, ainsi que des pratiques et conduites à risque. La deuxième partie explique les fondements des stratégies d'intervention et les mesures de surveillance épidémiologique et sanitaires qui ont permis d'améliorer les conditions d'hygiène et d'agir sur les carences primaires responsables de la prévalence de ces pathologies.
\end{abstract}

\section{Abstract}

This paper examines the social etiology of poverty-related infectious diseases prevalent in Spain and Italy in the first half of the twentieth century. Using an ecological model of health determinants, we analyze an explanatory framework consisting of the interaction between infectious agents, individuals vulnerable or susceptible to illness and unhealthy environments that facilitate contagion, on the one hand, and the action of fomites and vectors and high-risk practices and behaviors on the other. In addition, we discuss the bases of the intervention strategies and epidemiological and health surveillance measures that made it possible to improve hygienic conditions and mitigate the primary deficiencies responsible for the prevalence of these diseases.

Au cours des premières décennies du $\mathrm{XX}^{\mathrm{e}}$ siècle, grâce aux progrès de la médecine et au développement de la pathologie sociale, les conditions socio-sanitaires et l'amélioration des conditions de vie de la population sont redevenues primordiales dans la lutte contre 
les pathologies de nature infectieuse ${ }^{1}$. Le développement, à la fin du XIX ${ }^{\mathrm{e}}$ siècle, de la microbiologie et la découverte des germes responsables de pathologies comme la tuberculose, ont remis en cause le principe de multifactorialité qui avait guidé l'approche à leur égard ${ }^{2}$ et ont relégué au second plan la dimension sociale de l'hygiène. Sans pour autant renier la portée des agents biologiques, les hygiénistes sociaux soulignèrent l'insuffisance du rapprochement biologique naturel et de la maladie, en lien avec les idées étiopathogènes d'origine environnementale qui avaient guidé la lutte contre l'épidémie ${ }^{3}$. Comme l'indiquait l'hygiéniste Antonio $\mathrm{Mallou}^{4}$, en traitant le problème sanitaire urgent que représentait le trachome en Espagne en 1935 :

Contre le trachome, tout comme dans la défense contre de pareilles calamités publiques, nous devons actuellement recourir à la médecine sociale [...] en la considérant tant dans ses bases scientifiques que dans ses applications individuelles et collectives [...] les relations réciproques de la santé des hommes face à leurs conditions de vie [...] les infections fléaux des peuples sont les maladies sociales [...] c'est pourquoi la Médecine nécessaire, la Médecine sociale, dépasse les limites du dispensaire, du laboratoire, de l'hôpital pour s'introduire, loin de la biologie, dans l'économie, dans la psychologie inclusive.

Et, rappelant Étienne Brunet et sa référence aux maladies sociales dans son texte « La lèpre, médecine expérimentale et médecine sociale », Mallou lui-même ${ }^{5}$ remarquait que :

Sa propagation, sa ténacité, son évolution [celle des maladies sociales] dépendent de la solidarité et de la densité du groupe humain, mais également du fait que la société est divisée en catégories ou classes très différentes les unes des autres par leurs moyens d'existence, c'est-à-dire par leurs moyens de résistance à la maladie. La médecine sociale prend en compte les riches et les pauvres et, le fait que pour lutter contre les maladies, il faut compenser ces inégalités.

Pour aborder les conditions environnementales des maladies infectieuses, il fallait revendiquer la dimension sociale de l'activité médicale ${ }^{6}$ :

Le médecin ne doit plus être juste un rédacteur mécanique de formules, un observateur froid de malades, un sceptique studieux et scolaire, à l'écart des luttes économiques et politiques, réticent à connaître la vie sociale, fermé aux aspirations les plus légitimes

1. Bernardino Fantini, « La storia delle epidemie, le politiche sanitarie e la sfida delle malattie emergenti », Idomeneo, 2014/17, p. 9-42, ici p. 17-20.

2. Jorge Molero Mesa, « La lucha antituberculosa en la España del primer tercio del siglo XX », dans $E l$ Centro Secundario de Higiene Rural de Talavera de la Reina y la Sanidad Española de su tiempo, dir. Juan Atenza Fernández, José Martínez Pérez, Talavera, Junta de Comunidades de Castilla La Mancha, 2001, p. 131-148.

3. Esteban Rodríguez Ocaña, Por la salud de las naciones. Higiene, microbiología y medicina social, Akal Ediciones, Madrid, 1993.

4. Antonio Mallou, Un problema sanitario de urgente resolución. Conferencia impartida en el Instituto Nacional de Sanidad, Madrid, Instituto Nacional de Sanidad, 1935, p. 29-30.

5. Ibid., p. 30-31.

6. Témoignage de 1908 de Giuseppe Tropeano, l'un des partisans de la médecine sociale en Italie, cité dans : Renato Malta, Storia delle parassitosi nelle zolfare siciliane, Palerme, Accademia delle Scienze Mediche di Palermo/Plumelia Edizioni, 2013, p. 94. Pour le développement du concept de « médicalisation sociale », voir Bernardino Fantini, « La storia delle epidemie,... », op. cit., p. 19. 
des classes qui ne sont pas aisées (celles-là même où grouillent de façon tumultueuse les âmes humaines en souffrance) mais il doit être un connaisseur patient et attentif de l'engrenage social, un guetteur perspicace et intelligent de toutes les manœuvres réalisées par les personnes et les classes, les autorités et les gouvernements. Il doit être un médecin, c'est-à-dire, un observateur studieux, passionné et constant. Il doit être une personne qui connaît la souffrance, les injustices, les péchés, les idéaux, les nécessités et les aspirations de la société dans laquelle il vit puisqu'il est impossible d'expliquer fidèlement n'importe quel fait humain et biologique sans connaître l'organisation humaine et (sans connaître) les lois qui régissent cette organisation.

Mettre fin au cercle vicieux de la pauvreté, de la misère, de la malnutrition et de la maladie qui entouraient ces pathologies devint une priorité pour les politiques sanitaires et leur prévalence fut considérée comme un indicateur des mauvaises conditions de vie et de l'état de santé dont souffrait la population affectée par celles-ci ${ }^{7}$.

Le contrôle des maladies infectieuses associées à la misère et à la pauvreté représentait un défi pour les politiques de santé mais offrait également l'opportunité d'améliorer les conditions de vie de la population touchée, de couvrir les besoins hygiéniques les plus basiques et de corriger ainsi les causes de son incidence. Dans le contexte de l'étiologie multifactorielle à l'origine de ces problèmes de santé, comme l'indiquait un rapport élaboré en 1938 par une Commission mixte de la Société des Nations chargée de traiter le problème de la nutrition depuis le point de vue de la santé publique, de l'agriculture et des politiques économiques, le défi consistait à garantir à l'ensemble de la population une alimentation suffisante, saine et équilibrée (sans doute un élément clé pour expliquer la prévalence des maladies infectieuses associées à la misère et à la pauvreté). Il offrait, en même temps, la possibilité de sortir du sous-développement et de la pauvreté à l'origine de la faim et de la malnutrition ainsi que de nombreuses maladies infectieuses ${ }^{8}$.

Il s'agissait d'un ensemble de maladies sociales qui répondaient à une étiologie multifactorielle pouvant être évitées et qui étaient une expression biologique de la pauvreté et de l'inégalité sociale ${ }^{9}$, comme cela est le cas de nos jours en ce qui concerne les « maladies tropicales négligées ${ }^{10}$ ou $~ «$ maladies de la pauvreté négligées ${ }^{11}$ selon la définition de l'OMS.

La condition de maladies sociales qu'il fallait attribuer aux pathologies infectieuses associées à la misère et à la pauvreté, était définie non seulement par les répercussions sanitaires, socio-économiques, politiques ou culturelles associées à leur impact, mais également par le fait d'être provoquées par la propre organisation sociale et par des

7. Esteban Rodríguez-Ocaña, « Medicine as a Social Political Science. The Case of Spain c. 1920 », Hygiea Internationalis, 6/2, 2007, p. 37-52 ; et Bernardino Fantini, « La storia delle epidemie... », op. cit., p. 14-17.

8. Frank G. Boudreau, H. D. Kruse, « Malnutrition. A Challenge and an Opportunity », American Journal of Public Health, 29/5, 1939, p. 427-433.

9. Comme le rappelle Nancy Krieger (« Glosario de epidemiología social », Revista Panamericana Salud Pública, 11/5-6, 2002, p. 480-490), le concept « expression biologique de l'inégalité sociale », essentiel pour l'épidémiologie sociale, a été évident dans la pensée épidémiologique depuis l'apparition de la discipline au début du XIX ${ }^{\mathrm{e}}$ siècle, même s'il n'a pas toujours porté ce nom, comme le montrent les recherches pionnières concernant les gradients socio-économiques et les effets de la pauvreté sur la mortalité, la morbidité et la taille, telles que celles réalisées par Louis René Villermé (1782-1863).

10. Organisation Mondiale de la Santé (OMS), First report on neglected tropical diseases. Working to overcome the global impact of neglected tropical diseases, Genève, Organisation Mondiale de la Santé, 2010.

11. Merril Singer, Nicola Bulled, «Interlocked infections : the health burdens of syndemics of neglected tropical diseases », Annals of Anthropological Practice, 36/2, 2012, p. 328-345, ici p. 328. 
conditions de vie misérables qui reflétaient une importante inégalité sociale ${ }^{12}$. Selon l'épidémiologiste espagnol Manuel Mezquita, concernant la lutte contre le trachome dans la province d'Almería ${ }^{13}$, il s'agissait de pathologies dont les graves conséquences constituaient « un problème de santé publique se répercutant sur le domaine social », comme l'a démontré le fait que « les pourcentages de personnes atteintes de trachome étaient plus élevés dans les familles de faible niveau social ${ }^{14}$. Les hygiénistes italiens Alessandro Lustig et Achille Sclavo, ainsi que le médecin provincial Michele Alivia, allaient dans le même sens concernant la campagne anti-malarique menée en Sardaigne ${ }^{15}$ :

Continuer d'accroître la base de cette lutte contre la malaria en Sardaigne et étudier le meilleur moyen de lutter contre la malaria, harmonieusement, sans dispersion des moyens financiers et de l'énergie morale pour la lutte contre la malaria, la tuberculose et l'alcoolisme. Les médecins, qui ont noblement collaboré avec nous, ont été les premiers à admettre la nécessité, en Sardaigne, d'une lutte organique unique pour diriger la lutte contre toutes les causes de maladies et de détérioration physique qui appauvrissent le pays et détruisent la force vitale.

Ces dernières années, grâce à un point de vue interdisciplinaire, nous avons traité la problématique posée, dans le cas espagnol et italien (et plus concrètement celui de la Sardaigne), par la présence endémique du trachome, sans doute l'une des pathologies qui résume le mieux le modèle explicatif des maladies infectieuses associées à la misère et à la pauvreté ${ }^{16}$. Plus récemment ${ }^{17}$, nous avons publié un travail commun dans lequel nous avons analysé le rôle du Comité d'Hygiène de la Société des Nations et d'autres organismes internationaux dans la lutte contre le trachome. L'infection par trachome avait pris une

12. Jorge Molero, « La lucha antituberculosa en la España... », op. cit., p. 133-135.

13. Manuel Mezquita López, « La lucha contra el tracoma en la provincia de Almería », Revista de Sanidad e Higiene Pública, 29, 1955, p. 481-512, ici p. 481.

14. Ibid., p. 486.

15. Alessandro Lustig, Michele Alivia, Achille Sclavo, Relazione sommaria della Campagna antimalarica condotta nella provincia di Sassari nel 1910: contributo alla conoscenza delle condizioni igieniche-sociali della Sardegna, Florence, Società tipografica fiorentina, 1911, p. 4.

16. María Eugenia Galiana-Sánchez, Angela Cremades Monerris, Josep Bernabeu-Mestre, « Sanitary campaigns against trachoma in Spain », dans Making a New Countryside. Health Policies and Practices in European History ca. 1860-1950, dir. Astrid Andresen, Josep Lluís Barona et Steven Cherry, Francfort-sur-le-Main, Peter Lang, 2010, p. 101-111 ; Paola M. Melis, Lucia Pozzi, « Trachoma, health conditions and social change in Sardinia », dans Making a New Countryside..., op. cit., p. 25-48 ; Josep Bernabeu-Mestre, María Eugenia Galiana-Sánchez, « Salud comunitaria y acción social en el control epidemiológico del tracoma infantil, España (1932-1939) », Asclepio, LXIII/2, 2011, p. 507-520 ; Josep Bernabeu-Mestre, María Eugenia Galiana-Sánchez, « Determinantes laborales y de género en la epidemiología del tracoma en la España contemporánea », dans $E l$ trabajo y sus riesgos en la época contemporánea : conocimiento, codificación, intervención y gestión, dir. Aaron Cohen, Barcelone, Icaria/Publicacions i Edicions de la Universitat de Barcelona (Collecció Història del Treball, Volumen 5), 2012, p. 87-117 ; Josep Bernabeu Mestre, María Eugenia Galiana-Sánchez, Angela Cremades Monerris, «Environment and health in a poverty-related disease : epidemiology of trachoma in contemporary Spain, 1925-1941 », História Ciências Saúde-Manguinhos, 20/4, 2013, p. 1605-1619 ; Paola M. Melis, Lucia Pozzi, «Una malattia della povertà : il tracoma infantile in Sardegna », dans Mortalità e stato di salute dalla nascita alla prima adolescenza. Indagini Micro in Italia (secoli XIX-XX), dir. Marco Breschi et Lucia Pozzi, Udine, Forum, 2013, p. 157-187.

17. Lucia Pozzi, María Eugenia Galiana-Sanchez, Josep Bernabeu-Mestre, « La cooperación internacional en la lucha contra el tracoma. El papel de la Sociedad de Naciones, 1923-1935 », dans Sanidad internacional y transferencia del conocimiento científico. Europa, 1900-1975, dir. Josep Lluís Barona, Ximo Guillem-Llobat, Valence, PUV, 2015, p. 45-66. 
telle importante qu'elle était considérée comme une véritable calamité internationale et exigeait une prophylaxie qui passait, entre autres, par l'amélioration des conditions de vie des populations infectées et par l'application des règles d'hygiène les plus basiques ${ }^{18}$.

$\mathrm{Au}$ cours des premières décennies du $\mathrm{XX}^{\mathrm{e}}$ siècle, le trachome était endémique dans de nombreuses régions d'Europe méditerranéenne. L'Espagne était l'un des pays les plus touché, avec une zone endémique qui s'étendait sur tout le littoral méditerranéen, et des taux de mortalité qui oscillaient entre 2 et 4 pour mille ${ }^{19}$. Dans le cas de l'Italie, le trachome était endémique dans la zone méridionale, 24 provinces étant concernées (trois dans la partie méridionale de la péninsule, huit en Sicile et trois de l'île de Sardaigne), et les taux de mortalité atteignaient 45 pour $1000^{20}$.

Dans la présente étude, d'après la perspective offerte par l'histoire actuelle et les conclusions du passé ${ }^{21}$, on vise à approfondir l'examen des déterminants qui expliquaient la prévalence qu'atteignirent en Espagne et en Italie au cours de la première moitié du $\mathrm{XX}^{\mathrm{e}}$ siècle, les maladies infectieuses associées à la misère et à la pauvreté et quelle fût l'influence du diagnostic de pathologies sociales sur les stratégies d'intervention communautaire qui permirent leur contrôle et leur éradication. Dans ce but, nous avons principalement utilisé les témoignages et les diagnostics de pathologies comme la malaria, la tuberculose, le trachome ou la lèpre rassemblés dans les mémoires et les rapports publiés par les hygiénistes et les autorités sanitaires espagnoles et italiennes pendant la période étudiée.

Ces initiatives étaient en accord avec le contexte international. Dans le cas espagnol comme italien, au cours des premières décennies du $\mathrm{XX}^{\mathrm{e}}$ siècle et, en particulier pendant

18. Ibid., p. 59.

19. Josep Bernabeu Mestre, María Eugenia Galiana-Sánchez, Angela Cremades Monerris, « Environment and health... », art. cit., p. 1607.

20. Vittorio Ruata, Francesco Campanella, Lo stato attuale di diffusione del tracoma nell'Italia meridionale e insulare, Rome, Istituto Poligrafico dello Stato, 1940 (l'enquête officielle fut conduite en 1939 dans les provinces méridionales et insulaires).

21. Il s'agit d'un dialogue entre le passé et le présent ou, plus concrètement, entre l'expérience européenne dans la lutte contre les maladies infectieuses associées à la pauvreté et le défi posé de nos jours par les maladies tropicales négligées, qui a été revendiqué dans des travaux traitant de la problématique actuelle de ces pathologies, comme c'est le cas pour le trachome : Anne-Marie Moulin, « La lutte contre le trachome, de l'arrière-garde à l'avant-garde », M/S : médecine sciences, 21/12, 2005, p. 1101-1105; Hugh R. Taylor, « Doyne Lecture : trachoma, is it history? », Eye, 23, 2009, p. 2007 - 2022, et Helen Hamilton, Yael Velleman, « Washing Away Blinding Trachoma Health promotion should engage all community members », dans Trachoma : Poverty affects eye health, numéro spécial de Vision and Development. Ophthalmology in Development Cooperation, $2013, \mathrm{n}^{\circ} 1$, p. 10-11. 
l'entre-deux-guerres ${ }^{22}$, la médecine sociale connut un important niveau de développement et d'institutionnalisation ${ }^{23}$, qui en fit l'un des éléments à l'origine des réformes et transformations que connurent les administrations sanitaires des deux pays ${ }^{24}$.

\section{Les déterminants des maladies de nature infectieuse associées à la misère et à la pauvreté}

Comme le démontre la figure 1, conformément au modèle écologique des déterminants de la santée ${ }^{25}$, le développement des maladies infectieuses d'étiologie sociale serait le résultat de l'interaction entre l'agent, l'hôte et l'environnement, ou comme l'expliquaient les épidémiologistes de la période de l'entre-deux-guerres traitant le problème socio-sanitaire engendré par des maladies telles que le trachome, l'interaction entre trois facteurs primaires « le virus (l'agent étiologique), le transmetteur (les mécanismes de contagion), et l'hôte (les personnes exposées au risque de contracter la maladie $)^{26} »$.

L'environnement serait déterminé par la misère, la pauvreté et les inégalités où l'insalubrité, la surpopulation/promiscuité et d'autres facteurs de risques associés joueraient un rôle fondamental pour expliquer la prévalence des maladies infectieuses d'étiologie sociale, comme ce fut le cas de la lèpre dans la localité de Porcuna, dans la province de Jaén ${ }^{27}$ :

Les conditions d'hygiène sont mauvaises puisque les maisons ne possèdent pas l'eau courante et que cet élément doit être rapporté depuis un réservoir central alimentant six fontaines réparties dans le village, où se forment généralement de longues files d'attente, car en été les fontaines ne fonctionnent que quelques heures par jour. L'évacuation des immondices s'effectue par les égouts, mais dans la majorité des habitations appartenant à de petits paysans, celle-ci se fait dans le poulailler ou dans l'étable. Les maisons sont celles des zones rurales [...] un grand nombre de familles, parmi lesquelles une grande partie sont touchées par la lèpre, ont la mauvaise habitude

22. Iris Borowy, «International Social Medicine between the Wars : Positioning a Volatile Concept », Hygiea Internationalis, 6/2, 2007, p. 13-35 ; Josep Lluís Barona, Josep Bernabeu-Mestre, La salud y el Estado. El movimiento sanitario internacional y la administración española (1851-1945), Valence, Publicacions Universitat de València, 2008

23. Dans le cas italien, en 1917, fut constituée à Rome la Societá Italiana di Medicina Sociale, présidée par Giuseppe Tropeano, éditeur de la revue Medicina Sociale, publiée à Naples en 1911 (Adalberto Pazzzini, « Origine e sviluppo dellla medicina sociale in Italia », Rome, Istituto Italiano di Medicina Sociale [IIMS], 1966). Dans le cas espagnol, on note la création en 1912 de l'Institut Mèdic-Social de Catalogne et la publication entre 1916 et 1920 de la revue La Medicina Social Española, en tant que moyen d'expression de la Sociedad Española de Higiene (Esteban Rodríguez Ocaña, « La salud pública en la España de la primera mitad del siglo XX », dans El Centro Secundario de Higiene Rural de Talavera de la Reina y la Sanidad española de su tiempo, éd. Juan Atenza Fernández et José Martínez Pérez, Talavera de la Reina, Junta de Comunidades de Castilla-La Mancha, 2001, p. 21-42.

24. Claudio Pogliano, « L'utopia igienista (1870-1920) », dans Storia d'Italia, Annali 7, Malattia e medicina, éd. Franco Della Peruta, Turin, Einaudi, 1984, p. 598-631 ; Esteban Rodríguez-Ocaña, « Medicine as a Social Political Science : The Case of Spain c. $1920 »$, Hygiea Internationalis, 6/2, 2007, p. 37-52.

25. Il s'agit d'envisager les déterminants de la répartition de la maladie et des inégalités sociales dans le domaine de la santé, depuis un point de vue dynamique, historique et écologique (Nancy Krieger, « Glosario de epidemiología social », op. cit.).

26. Manuel Mezquita López, «El problema sanitario-social del tracoma. I Consideraciones epidemiológicas », Revista de Sanidad e Higiene Pública, XLII/5-6, 1968, p. 275-290.

27. Antonio Beltrán Alonso, « Epidemiología de la lepra en la provincia de Jaén », Memoria del VI Congreso Internacional de Leprología, Madrid, 1953, p. 977-1006, ici p. 1000. 
Fig. 1 -

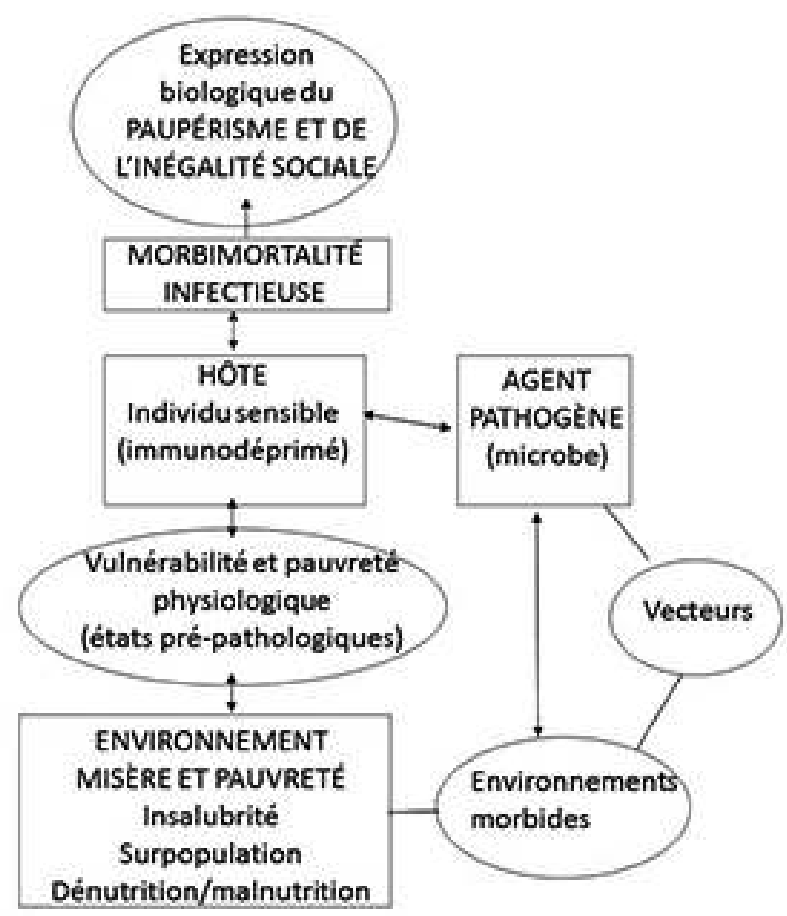

Source : élaboration des auteurs.

de dormir à plusieurs dans le même lit, regroupant en outre plusieurs lits dans la même pièce.

Dans le cas de l'hôte (les personnes susceptibles d'être infectées), sa vulnérabilité s'expliquerait principalement par la «pauvreté physiologique ${ }^{28}$ reflétée par les états prépathologiques que présentaient les individus vivant dans de tels environnements. Comme l'indiquait l'hygiéniste Luigi Maggiore en traitant le problème du trachome ${ }^{29}$, « la misère qui conduit à la pauvreté de l'alimentation et à la promiscuité qui facilite la contagion », expliquait les comorbidités qui caractérisaient ces contextes ${ }^{30}$ :

28. Le concept de pauvreté physiologique et les contextes de misère et d'inégalité sociale qui l'expliquaient, a été développé plus tard par les études d'épidémiologie sociale et plus concrètement avec la formulation du concept d' « embodiment » (Nancy Krieger, George Davey Smith, «'Bodies Count'and Body Counts : Social Epidemiology and Embodying Inequality », Epidemiologic Reviews, 26, 2004, p. 92-103).

29. Paola M. Melis, Lucia Pozzi, « Una malattia della povertà : il tracoma infantile in Sardegna », art. cit., p. 181-182.

30. Luigi Maggiore, Per combattere il tracoma nella città e nella provincia di Sassari. Problemi da risolvere e provvedimenti urgenti da adottare, Sassari, Comitato sassarese per la lotta contro il tracoma/Tip. Libreria Italiana e Straniera, 1926, p. 3. 
La lutte contre le trachome ne doit pas cibler uniquement les maladies oculaires. Le trachome est un puissant allié des organismes physiologiquement faibles à cause des tares de constitution, de la tuberculose, de la diathèse lymphatique, de la scrofulose, auxquelles s'ajoute également la malaria. Maladies qui ont fait leur apparition suite à une nutrition insuffisante et qui sont particulièrement prédisposées aux possibilités infinies de contagion liées au manque d'hygiène personnelle et familiale.

La malnutrition est apparue comme l'un des facteurs clés pour expliquer la prévalence de ces pathologies. De leur côté, les maladies infectieuses aggravaient la malnutrition et contribuaient à accroître la vulnérabilité des personnes affectées ${ }^{31}$, qui devenaient des hôtes susceptibles de développer la maladie infectieuse. De plus, cette dernière possibilité était accrue par le risque supérieur de contagion tant directement qu'indirectement (par des fomites et des vecteurs), qu'impliquaient l'insalubrité, la surpopulation et la promiscuité qui, comme cela a été indiqué, accompagnaient les situations de misère et de pauvreté. Comme pour la tuberculose, « la maladie ne se développait que si le sujet prédisposé était soumis à une série de causes qui permettaient le développement du germe (tuberculeux ${ }^{32}$ » ou comme il a été indiqué à propos du rôle de la diète dans le développement de la lèpre ${ }^{33}$ :

La malnutrition est l'un des facteurs les plus importants pour réduire la résistance. Néanmoins, la malnutrition est toujours accompagnée de facteurs importants pour la propagation de la maladie tels que l'absence de conditions d'hygiène, la promiscuité, etc., qui rendent difficile le diagnostic de la dénutrition.

Il en allait de même avec le trachome et le rôle de la nutrition quant aux conditions de l'hôte et aux possibilités de contagion ${ }^{34}$ :

Tout le monde ne le contracte pas (le trachome), car outre la virulence et le nombre de germes, interviennent des facteurs relatifs à l'environnement dans lequel vivent les individus sensibles (surpopulation, promiscuité, etc.) et à leur état de nutrition. Il ne faut pas oublier que le trachome est une maladie sociale, car les circonstances de ce type influent sur sa diffusion.

En ce qui concerne les micro-organismes (les agents) responsables des maladies infectieuses associées à ces environnements, contrairement à une approche traditionnelle où les maladies infectieuses sont traitées de façon isolée (sans prendre en compte les scénarios de comorbidité et indépendamment des contextes sociaux présents), il semble intéressant de traiter les interactions synergiques produites au sein d'environnements comme ceux associés à la misère et à la pauvreté, et les conséquences de cette interaction sur l'évolution, la diffusion, la gravité et le pronostic. Conformément au concept de pathocénose formulé

31. Gerald T. Keusch, « The History of Nutrition : Malnutrition, Infection and Immunity », Journal of Nutrition, 133/1, 2003, p. 336S-340S. Sur le concept de la vulnérabilité et son utilité explicative, voir Patrice Bourdelais, «Qu'est-ce que la vulnérabilité ? », Annales de démographie historique, 110, 2005/2, p. 5-9.

32. Jorge Molero, « La lucha antituberculosa en la España... », art. cit., p. 135.

33. Antonio Beltrán Alonso, « Epidemiología de la lepra en la provincia de Jaén... », art. cit., p. 995.

34. Manuel Mezquita López, « El problema sanitario-social del tracoma... », art. cit., p. 276. 
par Grmek ${ }^{35}$ ou avec la perspective de « syndémie » proposée par Merrill Singer, ${ }^{36}$ il s'agit de connaître les conditions sociales et comment celles-ci contribuent à l'interaction entre les maladies et à leur diffusion ${ }^{37}$. Comme l'expliquait un rapport sur la distribution spéciale du trachome dans la province d'Almería ${ }^{38}$, la principale prévalence était liée aux « quartiers où le niveau de vie est faible et dans lesquels on retrouve tous les facteurs qui conditionnent la diffusion des maladies de type social (tuberculose, trachome, etc.) ».

Il s'agissait de pathologies qui partageaient de nombreux facteurs de risque et qui nécessitaient, par conséquent, une action coordonnée au moment de les $\operatorname{traiter}^{39}$ :

La lutte contre le trachome, contre la malaria, contre la tuberculose, contre l'alcoolisme et les nombreuses affections cutanées liées à la saleté doit être, au moins pour le moment, un travail organique et unitaire ; il ne doit pas être fragmenté en tentatives partielles et unilatérales, souvent coûteuses et peu efficaces.

Comme le rappelait Domenico Pietri à propos de la lutte contre la tuberculose dans l'Italie fasciste et de son statut de maladie sociale ${ }^{40}$ :

Le fait que la tuberculose ne soit quasiment jamais isolée mais qu'elle soit étroitement liée à la présence d'autres maladies sociales aussi graves, constitue une donnée de base du problème de la tuberculose que nous ne devons absolument pas négliger afin de ne pas courir le risque, comme ce fut le cas pendant la période fasciste, de traiter uniquement la tuberculose tout en négligeant les autres maladies.

Ci-après, afin d'illustrer la dynamique du modèle explicatif qui vient d'être exposé, sont présentés quelques exemples de l'interaction des déterminants qui expliquaient la prévalence de maladies infectieuses d'étiologie sociale associées à la misère et à la pauvreté, le rôle que jouèrent les environnements morbides comme l'habitat domestique, ou le diagnostic et les propositions qui, à partir de ce modèle explicatif, ont été formulés par les hygiénistes chargés de ces pathologies et de leurs causes, pour prévoir leur prévention et leur éradication.

\section{Le facteur environnemental dans le développement des maladies infectieuses associées à la pauvreté : l'exemple de l'habitat domestique}

Dans les environnements domestiques et familiaux miséreux et pauvres, le faible niveau d'instruction et de connaissances en matière d'hygiène et de santé s'ajoutait à l'insalubrité

35. Sur la signification et l'évolution du concept de pathocénose et la question de comorbidité depuis la perspective de la population, voir Bernardino Fantini, « La storia delle epidemie,... », art. cit., p. 29-31.

36. «Un point de départ pour l'analyse syndémique est un examen des conditions sous-jacentes et les relations sociales, leurs causes et leurs contextes, ainsi que les facteurs naturels et anthropiques environnementaux qui facilitent le regroupement et l'interaction des différentes maladies... » : Merril Singer, Nicola Bulled, «Interlocked infections... », art. cit., p. 329.

37. «Un cercle vicieux impliquant une spirale où entrelacent pauvreté et la maladie et qui est aggravée par le développement des multi-maladies syndémiques » (ibid., p. 332).

38. Manuel Mezquita López, « La lucha contra el tracoma... », art. cit., p. 495-496.

39. Alessandro Lustig, Michele Alivia, Achille Sclavo, Relazione sommaria della Campagna antimalarica..., op. cit., p. 82.

40. Domenico Pietri, « La lotta antitubercolare nell'Italia fascista », dans Malattia e medicina, Annali della storia d'Italia, 7, dir. Franco della Peruta, Turin, Giulio Einaudi editore, 1984, p. 955-1021, ici p. 955-956. 
et à la surpopulation. Les conditions des habitations et l'absence de prestations de cellesci (par exemple, cycle de l'eau, chauffage, ventilation, cuisine, etc.), la distribution des espaces domestiques, la présence d'animaux domestiques et la cohabitation avec ceux-ci, les styles et les habitudes de vie, y compris alimentaires, sont quelques-uns des éléments qui expliqueraient les facteurs et les pratiques à risque (comme, par exemple, le partage d'un même lit par plusieurs personnes) rendant les composants du groupe familial vulnérables à la maladie infectieuse ou qui permettraient de comprendre les interactions entre les différentes pathologies se produisant dans ces environnements (depuis le partage de mécanismes et opportunités de contagion à l'action en régime de comorbidité). La précarité à laquelle devait faire face la majorité des familles touchées par les maladies infectieuses d'étiologie sociale, se traduisait, par exemple, par une absence de nécessaires de toilette individuels et la fréquente contamination de ceux existants, favorisant la contagion de l'individu malade à l'individu sain, comme le reflète le témoignage des médecins italiens Lucio Pintus et Bachisio Latte ${ }^{41}$, faisant référence aux environnements dans lesquels devaient retourner les enfants atteints de trachome alors qu'ils avaient participé aux colonies scolaires :

Il est facile de deviner quel sera l'avenir de ces enfants inscrits dans les colonies qui, quand ils sont sains du trachome et en meilleur état du point de vue biologique, retournent dans leurs pays, souvent sans système d'évacuation des eaux usées, ni d'égouts, sans écoles suffisantes, au sein de leurs familles où cohabitent les personnes saines et malades, sans possibilité d'isolement, dans ces environnements touchés par la pire des misères, sans services d'hygiène, où l'on dort tous dans le même lit, où tout le monde fait sa toilette dans la même bassine et se sèche avec le même chiffon.

La gravité des déficiences présentées par les habitats domestiques où se développaient ces pathologies, se reflète dans des expressions comme celles utilisées par Domenico Pietri faisant référence, dans le cas de la tuberculose, au «drame des habitations ${ }^{42}$ :

Plus les habitations s'éloignent de la définition même d'habitation, plus elles ressemblent à d'horribles taudis humides et malsains, sans air ni lumière, plus pauvres, de construction plus fragile et plus la population se montre prédisposée à y vivre et pire seront ses vêtements, son alimentation, sa culture de l'hygiène, etc.

Des pathologies comme la lèpre ${ }^{43}$ ou le trachome étaient considérées comme des « infections éminemment familiales » puisque, contrairement aux autres affections transmissibles, «elles nécessitent des contacts très intimes et répétés », de sorte que lorsque le germe arrivait dans un foyer sa diffusion ne tardait pas à se produire «pour peu que l'antihygiène régna dans la maison ${ }^{44}$. Le témoignage de l'hygiéniste italien Salvatore

41. Lucio Pintus, Bachisio Latte, « Alcuni aspetti delle condizioni igienico-sanitarie ed economico-sociali dei tracomatosi in provincia di Nuoro, Nota III », L'Igiene Moderna, 11-12, 1960, p. 720.

42. Domenico Pietri, « La lotta antitubercolare nell'Italia fascista », art. cit., p. 962.

43. Dans les travaux sur l'épidémiologie de la lèpre, on parte de familles de « lazarinos », pour faire référence à la concentration de cas qui se produisait habituellement dans certains groupes familiaux, voir Egea Bueno, «Exploración de familiares y convivientes de enfermos lazarinos (Censo de convivientes de la provincia de Granada. Lesiones iniciales) », Memoria del VI Congreso Internacional de Leprología, Madrid, 1953, p. 10071013, ici p. 1010.

44. Manuel Mezquita López, « La lucha contra el tracoma... », art. cit., p. 491-493. 
Collari, faisant référence au problème de la tuberculose dans l'Italie rurale, démontre la détérioration en matière d'hygiène que pouvaient atteindre les habitats domestiques ${ }^{45}$ :

L'environnement de vie dans la famille et dans le village est le plus antihygiénique imaginable. La cuisine typique des petits villages de Sardaigne, tout particulièrement dans la montagne, possède un foyer carré creusé au centre du séjour, c'est l'endroit où se déroule toute la vie intime de la famille. On y mange, on y fait les tâches domestiques, on y reçoit, on y passe son temps et on y dort. Généralement, le sol de ces pièces est fait de terre, meuble ou battue, aménagée de pavés. Lors des repas, la famille se réunit autour du foyer, sur lequel est posé le repas dans des « tazeris », récipients concaves en bois, dans lesquels on se sert assis « à la turc ». C'est là, autour du foyer, surtout en hiver, qu' on se retrouve pour parler et boire avec les amis et connaissances, dans le même récipient «sa zucca », tout en fumant et crachant sur le sol. C'est toujours à ce même endroit que les enfants jouent sur le sol et que les adultes, tout particulièrement les femmes, passent leur journée. En fin de compte, il s'agit souvent d'une pièce unique où toute la famille passe également la nuit, couchée sur des tapis ou de simples peaux de chèvre ou de mouton, posés directement sur la terre nue.

Comme il a été signalé dans une étude sur l'épidémiologie de la lèpre à Jaén ${ }^{46}$, une province où le chômage ouvrier était « l'explication fondamentale de la misère et du faible niveau de vie de la majeure partie de la population rurale ${ }^{47}$, l'état de la maison avait « une importance extraordinaire dans la propagation de la maladie ${ }^{48}$. L'incidence élevée de la lèpre pouvait s'expliquer, en grande partie, par les mauvaises conditions hygiénicosanitaires rencontrées dans la majorité des maisons des classes ouvrières (manque d'eau, mauvaises habitudes de nettoyage, etc.), et en particulier celles des malades de la lèpre, où on notait une surpopulation, facteur de risque car il facilite la cohabitation intime et la contagion directe, mécanisme de transmission le plus courant ${ }^{49}$ :

L'état de la maison revêt une importance fondamentale dans la propagation de la maladie puisqu'il s'agit de l'élément qui conditionne principalement la promiscuité [...] une seule pièce servant de chambre, une entrée ou vestibule, servant habituellement de salle à manger et une cour ouverte abritant dans un coin une décharge et dans l'autre un petit abri qui sert de cuisine.

Dans l'ensemble de la province, sur 152370 maison recensées, 26,3\% de celles-ci étaient considérées directement comme défectueuses du point de vue de l'hygiène, 42,5\% étaient défectueuses et seulement $32 \%$ étaient classées comme hygiéniques ${ }^{50}$.

45. Salvatore Collari, La tubercolosi rurale. Cause della diffusione della tubercolosi nelle campagne e modi di evitarla. Con confronti internazionali, Rome, Stabilimento Tipografia Europa, 1934, p. 72-73.

46. Antonio Beltrán Alonso, « Epidemiología de la lepra en la provincia de Jaén... », art. cit., p. 981.

47. Ibid., p. 979.

48. Ibid., p. 981.

49. Ibid.

50. Ibid. 
Dans les localités comme Torreperogil ${ }^{51}$, l'un des principaux foyers de lèpre de la province, le mode de vie courant était le « logement de plusieurs familles dans une même maison, disposant chacune d'une seule pièce ». Il n'existait pas d'égouts et, dans la plupart des maisons des familles affectées, les immondices étaient évacuées dans le poulailler ou dans des fosses. Le ravitaillement en eau se faisait dans des fontaines publiques et avait tendance à se raréfier en été.

Dans la province voisine de Grenade, l'habitat domestique abritant les malades de la lèpre, présentait également d'importantes déficiences, y compris au niveau de l'alimentation $^{52}$ :

La grande majorité des malades vivent dans des maisons dont les conditions d'habitabilité sont mauvaises : promiscuité, surpopulation et manque d'hygiène, proximité des animaux domestiques et d'élevage, volailles, présence abondante de puces, punaises et toute sorte d'insectes [...] l'alimentation est généralement déficiente en quantité et qualité, principalement constituée de pommes de terre, poisson, légumes secs, légumes et pain, avec très peu de viande.

On notait les mêmes conditions dans d'autres régions d'Europe méditerranéenne concernées par une prévalence élevée des maladies infectieuses associées à la misère et à la pauvreté, comme c'était le cas de l'île italienne de Sardaigne ${ }^{53}$ :

Il est facile d'imaginer que, lorsque cinq, six ou sept personnes cohabitent dans une même pièce et que souvent la plupart sont des enfants, certaines soient atteintes de tuberculose ou de trachome. Il est facile d'imaginer comment ces maladies se diffusent rapidement et facilement. [...] Le rapport rédigé par la représentante de l'Unione donne sarde déclare : pendant la journée, tout se déroule dans ces quelques mètres carrés : l'enfant qui grignote un morceau de pain, assis sur le sol ; l'homme qui souhaite se reposer après une journée de travail difficile (quand il travaille puisque la majorité des chefs de famille est au chômage partiel ou total); la femme qui doit préparer un déjeuner frugal ou repriser le linge troué. Entre huit et dix personnes vivent dans ces quelques mètres carrés.

Dans le cas du trachome ${ }^{54}$, maladie qui comme la lèpre est considérée comme une «contagion torpide, qui nécessite pour être propagée des contacts répétés et longs », le mécanisme le plus fréquent était direct, produit par « la sécrétion conjonctivale qui contamine les mains du malade » et au sein des familles « où les mères allaitantes et les enfants les plus jeunes » étaient contaminés « par les pères et frères et sœurs plus âgés ». Dans le cas de contagion directe, également dans le cadre familial, la responsabilité était attribuée aux vecteurs passifs et plus concrètement à l'utilisation d'objets d'usage commun (serviettes, mouchoirs, etc.) contaminés par la sécrétion conjonctivale des malades.

Les écoulements conjonctivaux étaient néanmoins beaucoup plus contagieux lorsqu'ils étaient mélangés à des « écoulements purulents ou catarrhaux propres aux conjonctivites

51. Ibid., p. 1004.

52. Egea Bueno, « Censo de lazarinos de la provincia de Granada (Mapa leprológico) », Memoria del VI Congreso Internacional de Leprología, Madrid, 1953, p. 1022-1025, ici p. 1025.

53. Atti Parlamentari, 1952, p. 40868-40869.

54. Manuel Mezquita López, « El problema sanitario-social del tracoma... », art. cit., p. 276. 
surinfectées caractéristiques de fin de printemps et fin d'été (saisonnières), écoulements qui servent d'éléments de conservation, de transport (en particulier via les mouches) et de contamination de la maladie $»^{55}$.

Le statut de réservoirs de maladie que présentaient ces habitats domestiques a été très bien illustré dans le témoignage du médecin et écrivain italien Carlo Levi ${ }^{56}$ :

Il y avait une multitude d'enfants. Dans cette chaleur, parmi les mouches et la poussière, ils sortaient de partout, entièrement nus ou vêtus de loques. Je n'avais jamais vu une telle misère, pourtant je suis habitué à voir tous les jours des dizaines d'enfants pauvres, malades et mal soignés, c'est mon travail. Mais jamais je n'avais imaginé voir ce que j'ai vu hier. J'ai découvert des enfants assis devant les portes des maisons, dans la saleté, sous un soleil brûlant, les yeux à demi-fermés et les paupières rouges et gonflées, des mouches se posant sur leurs yeux tandis que les enfants restaient immobiles sans les effrayer ou les chasser avec les mains. Oui, les mouches se promenaient sur leurs yeux et ils n'avaient pas l'air de les sentir. C'était le trachome. Je savais qu'il existait dans cet endroit mais le voir comme ça, parmi la saleté et la misère, c'est une chose différente. J'ai rencontré d'autres enfants aux visages ridés comme des vieux, squelettiques à cause de la faim, les cheveux pleins de poux et de croûtes. La plupart d'entre eux avaient un ventre gonflé, énorme et le visage jaune et décharné par la malaria.

\section{L'action prophylactico-sociale et l'intervention communautaire dans la lutte contre les maladies infectieuses associées à la pauvreté}

Comme on l'a vu dans la partie précédente, l'habitat domestique jouait un rôle fondamental dans le développement des maladies infectieuses d'étiologie sociale, tout en offrant un cadre parfait pour l'application des mesures préventives qui exigeait, selon le modèle explicatif des déterminants, le contrôle et l'éradication de ces pathologies.

Un grand nombre de maladies infectieuses associées à la misère et à la pauvreté étaient considérées comme familiales car elles étaient plus prévalentes dans les familles à risque. Comme l'indique Manuel Mezquita López ${ }^{57}$, dans le cas du trachome, « chaque malade rencontré représente avec certitude un foyer familial », et par conséquent, " la nécessité de se rendre dans les domiciles respectifs pour découvrir les malades qui y sont probablement présents ». Ce même auteur, lors de la lutte contre le trachome dans la province espagnole d'Almería, notait ce qui suit ${ }^{58}$ :

Il faudra prendre en compte que l'écolier souffrant de trachome représente une phase de la contagion au sein de sa famille, ou plutôt selon nous des unités familiales, c'est-à-dire que chaque écolier malade doit toujours être considéré comme un foyer d'infection à son domicile plus ou moins dense selon le nombre de membres de la famille affectée.

55. Manuel Marin-Amat, « La lucha antitracomatosa en España y sus satisfactorios resultados. Entrevista en Radio Nacional de España, con el Dr. Marin-Amat », Archivos de la Sociedad Oftalmológica Hispano-Americana, 18/9, 1958, p. 992-997, ici p. 992.

56. Carlo Levi, Cristo si è fermato a Eboli, 1945, cité dans l'édition de 2010 (Turin, Edizione Einaudi, p.79).

57. Manuel Mezquita López, «El problema sanitario-social del tracoma... », art. cit., p. 277.

58. Manuel Mezquita López, « La lucha contra el tracoma... », art. cit., p. 492. 
La lutte contre les agents impliquait l'élimination des sources d'infection qui, dans le cas de maladies comme le trachome ou la lèpre, nécessitait le « recensement des malades », tout en effectuant dans la plupart des cas des «identifications en série des enfants en âge scolaire et des travailleurs agricoles et industriels ${ }^{59}$, ce qui engendrait généralement des pourcentages élevés de cas positifs, comme on peut le noter dans le tableau 1.

Tab. 1 - Résultats de l'identification des scolaires et ouvriers dans les zones très infectées par le trachome dans la province d'Almería (1952)

\begin{tabular}{|c|c|c|}
\hline Scolaires identifiés & Positifs & Pourcentage \\
\hline 7433 & 2923 & 39,3 \\
\hline Ouvriers identifiés & Positifs & Pourcentage \\
\hline 5706 & 1407 & 24,6 \\
\hline
\end{tabular}

Source : Manuel Mezquita, "La lucha contra el tracoma... », 1955, op. cit., p. 490.

Une fois les foyers familiaux détectés, il fallait intervenir sur les éléments de l'habitat domestique qui facilitaient la contagion, en essayant de mettre en place des mesures comme « le nettoyage des serviettes de toilettes, mouchoirs et, en général, de tous les objets d'utilisation courante, en évitant le contact entre les yeux et tout ce qui pourrait être contaminé », ainsi qu'en essayant de favoriser l'utilisation individuelle des objets de toilettes et d'hygiène personnelle ou l'utilisation d'eau pour ces besoins, «en appliquant les précautions nécessaires pour ne pas être contaminé par ce mécanisme ${ }^{60}$. Sans oublier néanmoins la lutte contre des vecteurs comme les mouches ${ }^{61}$ :

Dans ce sens, le nombre de mesures prises pour réduire les risques de contagion massive en fonction de l'époque de l'année est très important et, étant donné que les mouches jouent un rôle pour véhiculer le germe à l'origine de ces conjonctivites, il ne faut pas oublier de désinsectiser systématiquement aux dates appropriées ainsi, en combattant ces insectes, on peut prévenir la diffusion des conjonctivites aiguës et, au passage, effectuer une prophylaxie anti-trachome.

Dans le cas de la lèpre, une autre des maladies infectieuses qui explique parfaitement le modèle explicatif que nous analysons, la prophylaxie se basait sur l'internement des malades contagieux et sur l'examen des foyers familiaux effectués par les dispensaires dermatologiques et d'hygiène sociale ${ }^{62}$. Une fois le recensement des malades effectué, on réalisait dans les dispensaires un travail « d'examen et de contrôle des malades et parents et cohabitants de ces zones où la morbidité était la plus élevée ${ }^{63}$. Les identifications périodiques et successives réalisées par les équipes mobiles, étaient essentielles pour le contrôle et l'éradication de la maladie ${ }^{64}$ :

59. Ibid., p. 484. Il existait tout un ensemble d'activités agricoles qui représentaient un facteur de risque pour la contagion par trachome (voir, pour le cas espagnol, le travail de Josep Bernabeu-Mestre et María Eugenia Galiana-Sánchez, « Determinantes laborales y de género en la epidemiología del tracoma... », art. cit.).

60. Manuel Mezquita López, «El problema sanitario-social del tracoma... », art. cit., p. 277.

61. Manuel Mezquita López, « La lucha contra el tracoma... », art. cit., p. 500.

62. Antonio Cordero Soroa, «El problema de la lepra en España (Control y profilaxis) », Memoria del VI Congreso Internacional de Leprología, Madrid, 1953, p. 922-976, ici p. 937.

63. Ibid., p. 938.

64. Ibid., p. 955. 
La longue période d'incubation de la lèpre [...] poussait à rechercher et examiner dans les foyers familiaux les cas douteux ou suspects [...] afin de repérer la maladie dès son commencement, moment où elle peut être facilement soignée et où il est possible d'éviter de former de nouveaux foyers de contagion, puisqu'il a été admis que les cas ignorés de contagion, sont la principale cause de propagation de la maladie.

Les recherches effectuées par les équipes mobiles se basaient sur des fiches familiales, qui outre le fait d'indiquer « les circonstances d'hygiène et conditions de la maison ainsi que la situation économique de la famille », regroupaient les données cliniques et socioéconomiques des malades (âge, profession ou résidences précédentes) et des parents et cohabitants, avec l'indication du lien de parenté, de la classe et du temps de cohabitation ${ }^{65}$.

Les activités prophylactiques ayant comme objet l'hôte ${ }^{66}$, avaient pour objectif, comme nous l'avons déjà précisé, d'élever le niveau de vie, ce qui impliquait une « amélioration de l'alimentation pour produire de meilleures défenses et une résistance plus élevée face à la maladie ${ }^{67}$, tout en améliorant les conditions des maisons, entre autres :

Finalement, tout ce qui est censé favoriser les conditions de vie entraînera une augmentation du nombre d'obstacles pour la propagation de l'infection, intimement liée au contexte socio-économique. Â ce point, selon de nombreux auteurs, sans mesures sanitaires strictes, le problème du trachome pourrait se résoudre si les problèmes sociaux sur lesquels il repose sont résolus.

Les dispensaires devinrent une pièce clé dans la lutte contre les maladies infectieuses d'étiologie sociale et dans les activités communautaires ${ }^{68}:$ "Nous insistons sur la mise en place d'unités de soins ambulatoires et de dispensaires à des fins sanitaires multiples et nous pensons que ces dispensaires devraient disposer de personnes chargées de la surveillance sanitaire, choisies parmi celles qui connaissent la population. »

Le travail de dépistage précoce, de diagnostic et de traitement effectué depuis les dispensaires, devait compléter les activités d'éducation sanitaire réalisées par les infirmières à domicile ${ }^{69}$, tout particulièrement aux domiciles des personnes affectées ${ }^{70}$ :

L'action intime au sein des familles offre des avantages pour notre action qui dérivent d'une clientèle très importante qui voit de ses propres yeux et acquiert sans se rendre compte des habitudes médico-hygiéniques et qui, sans résistance dans un premier

65. Egea Bueno, « Censo de lazarinos de la provincia de Granada... », art. cit., p. 1008.

66. Manuel Mezquita López, « El problema sanitario-social del tracoma... », art. cit., p. 277.

67. Le traitement du « facteur alimentaire, maillon important dans la chaîne du trachome » était mis en place dans la lutte contre le trachome dans le domaine scolaire, par le biais des cantines scolaires qui étaient développées dans les localités où existaient les classes spéciales ou les écoles pour malades du trachome (Manuel Mezquita López, « La lucha contra el tracoma... », art. cit., p. 501).

68. Alessandro Lustig, Michele Alivia, Achille Sclavo, Relazione sommaria della Campagna antimalarica..., op. cit., p. 82.

69. Manuel Mezquita López, « La lucha contra el tracoma... », art. cit., p. 500. Concernant le rôle des dispensaires dans le développement des activités de santé communautaire : Josep Bernabeu-Mestre, « Transición sanitaria y evolución de la medicina (diagnóstico, profilaxis y terapéutica) 1885-1942 », Revista de Demografía Histórica, 16/2, 1998, p. 15-38, ici p. 34-35, et Bernardino Fantini, « La storia delle epidemia... », art. cit., p. 14-17.

70. Manuel Marin-Amat, « La lucha antitracomatosa en España y sus satisfactorios resultados... », art. cit., p. 910 . 
temps puis avec envie, nous ouvre la porte de leur maison. C'est la clé [...] du succès de la santé officielle à domicile.

Ces activités ont été complétées par la propagande sanitaire diffusée par la radio ${ }^{71}$, les affiches $^{72}$ ou les débats et conférences d'information ${ }^{73}$ :

Nous sommes convaincus qu'outre le fait d'avoir permis de réduire le nombre de malades, quelque chose de difficile à mesurer ou enregistrer statistiquement mais qui, cependant, revêt une importance extraordinaire dans la lutte contre les maladies sociales a été obtenu. Nous faisons référence à l'augmentation de l'éducation sanitaire qui, sans aucun doute, s'est répandue dans le public lorsque celui-ci a saisi la gravité du problème et vérifié les circonstances favorables dérivées de la campagne développée. L'inquiétude et l'intérêt montrés par les masses populaires, en tant qu'expression de leur foi dans la médecine préventive, ont été correctement exploités [...] la diffusion des connaissances hygiéniques élémentaires a permis d'élever le niveau culturel et ainsi de contribuer à créer un environnement qui favorise et complète nos plans sanitaires.

Comme établi dans les directives émises par le Comité d'experts sur le trachome de l'OMS ${ }^{74}$, le contrôle des maladies infectieuses d'étiologie sociale passait par « le dépistage et le traitement des malades », ce qui signifiait la suppression des sources de contagion et l'élimination des «éventuels agents vecteurs » via le contrôle des facteurs qui facilitent la contagion et par une éducation sanitaire complétée par des «mesures d'assainissement et sociales correspondantes ». Le bilan réalisé lors de la lutte contre le trachome dans le cas espagnol ${ }^{75}$ renforçait ces considérations. Bien que "l'assistance des ressources thérapeutiques modernes (sulfamides par voie orale et crème d'auréomycine par application locale) » ait fourni des résultats satisfaisants, ils auraient été meilleurs « en agissant sur un fond d'amélioration sociale sur toutes les manifestations d'hygiène, bien-être économique et éducation de la classe pauvre, principale victime du trachome ».

Il s'agissait de la même stratégie défendue par les hygiénistes italiens de l'entre-deuxguerres $^{76}$ :

Dans les familles abritant des personnes contaminées, il faudra mettre en place des normes d'hygiène personnelle extrêmement rigoureuses et, simultanément, persuader que le nettoyage approprié des mains avec de l'eau et du savon, ainsi que l'utilisation personnelle de mouchoirs, serviettes et coussins sont des moyens largement suffisants pour éviter la contagion. Malheureusement, il est inutile de prêcher la propreté à des

71. Voir pour le cas espagnol : Àngela Bernabeu-Peiró, « La divulgación higiénico-sanitaria en los inicios de la radio española (1933-1957) », Historia y Comunicación Social, 20/1, 2015, p. 223-240.

72. Ramón Castejón Bolea, Enrique Perdiguero Gil, José Luis Piqueras Fernández, Las imágenes de la salud : cartelismo sanitario en España (1910-1950), Alicante, Instituto Alicantino de Cultura Juan Gil-Albert/Consejo Superior de Investigaciones Científicas, 2012.

73. Manuel Mezquita López, « La lucha contra el tracoma... », art. cit., p. 502.

74. Ibid., p. 498-499. p. 906.

75. Manuel Marin-Amat, « La lucha antitracomatosa en España y sus satisfactorios resultados... », art. cit.,

76. Luigi Debenedetti, « Diffusione del tracoma nella città di Sassari. Rilievi statistici e considerazioni profilattiche », Studi Sassaresi, serie II, volume II, fasc. 1, Sassari, Tipografia Giovanni Gallizzi, 1923, p. 16. 
personnes regroupées dans des environnements malsains, sans sous-vêtements, sans savon et même sans eau. Pour cette raison, la lutte contre le trachome est également un problème social, le problème de la misère ; plus le niveau de vie est élevé, moins il y a de contamination. A Sassari, la solution au problème de l'eau potable et des maisons populaires via l'expulsion des logements insalubres et, surtout, une meilleure prospérité des catégories sociales les plus basses, contribuera de manière décisive à empêcher la propagation continue et inévitable de l'infection aux générations suivantes au sein d'une même famille. On observe des familles, qui sont devenues des environnements où règnent la maladie et la misère et qui se retrouvent dans l'impossibilité d'empêcher la contagion aux enfants au fur et à mesure qu'ils viennent au monde. Pour ces personnes, les conseils, les avertissements et les instructions ne servent à rien. On ne pourra venir à bout de ces foyers infectieux qu'en assainissant l'environnement et en améliorant leurs conditions de vie.

En lien avec ces dernières considérations, comme le rappelait l'épidémiologiste Manuel Mezquita López ${ }^{77}$, s'appropriant les mots de l'hygiéniste italien Luigi Maggiore, pour éradiquer des maladies comme le trachome, il ne suffisait pas d'utiliser des traitements spécifiques mais il était nécessaire d'élever le niveau de vie de la population affectée et de développer des programmes d'éducation sanitaire efficaces, puisque «l'un des facteurs les plus importants pour lutter efficacement contre toute maladie sociale consiste à développer au sein du peuple la conscience de la gravité des maux et la connaissance des moyens pour les combattre ». Comme l'indiquait l'ophtalmologue espagnol Manuel Marín Amat en établissant un bilan des résultats de la lutte contre le trachome en Espagne $^{78}$ :

En général, le trachome est synonyme de saleté, de misère et d'absence de culture, de sorte que les peuples qui en souffrent, à un niveau accentué, sont les plus pauvres et les plus en retard au monde, aussi la prophylaxie repose donc sur la propreté, le bien-être économique et l'éducation.

Selon les mots des hygiénistes italiens Alessandro Lustig, Michele Alivia et Achille Sclavo $^{79}$, la clé pour traiter le problème de la malaria en Sardaigne résidait dans une action intégrale capable d'améliorer les conditions d'hygiène à travers le développement économique et social :

Améliorer les conditions d'hygiène générales et activer conjointement et de toutes les façons possibles le progrès économique et social. La lutte contre la malaria n'est qu'une facette du problème excessivement complexe de la régénération de la Sardaigne. Considérer la question d'un point de vue trop unilatéral serait une erreur : cela reviendrait à éclipser la question. La malaria est seulement le résultat de conditions économico-sociales et hygiéniques générales du lieu et la maladie, à son tour, aggrave encore la situation.

77. Manuel Mezquita López, «El problema sanitario-social del tracoma... », art. cit., p. 283.

78. Manuel Marin-Amat, « La lucha antitracomatosa en España y sus satisfactorios resultados... », art. cit., p. 993.

79. Alessandro Lustig, Michele Alivia, Achille Sclavo, Relazione sommaria della Campagna antimalarica..., op. cit., p. 82. 


\section{Conclusions}

Les résultats ont souligné l'utilité de se référer au modèle explicatif des maladies infectieuses associées à la misère et à la pauvreté, puisqu'il permet de démontrer l'étiologie sociale de ces pathologies ainsi que les principes fondamentaux qui élaborent les stratégies d'intervention permettant de traiter les facteurs déterminants à l'origine de leur prévalence.

Il souligne, dans un premier temps, le rôle des environnements morbides comme l'habitat domestique et la condition de «maladie familiale» qui reliaient de nombreuses infections d'étiologie sociale. Ensuite, il présente les états pré-pathologiques, en référence au risque que représentait pour la plupart des maladies infectieuses, la « pauvreté physiologique » que présentaient bon nombre de malades et qui était associée à la misère conduisant à la « pauvreté de l'alimentation » et à la promiscuité favorisant la contagion. Il s'agissait, en fait, de tout un ensemble de carences primaires, depuis celles associées à l'assainissement et la malnutrition jusqu'à celles de nature hygiénique et celles associées à des pratiques à risque qu'entraînaient un faible niveau d'instruction et l'absence de connaissances hygiénico-sanitaires.

Pour faire face à cette problématique, on développa un modèle d'intervention préventive basé sur les budgets qui définissaient la santé communautaire de l'entre-deux-guerres comme modalité d'exercice sanitaire de la médecine sociale et de la santé publique. Les politiques de santé mises en œuvre s'appuyaient sur le modèle du dispensaire et sur l'application des mesures de surveillance épidémiologique (détection précoce, recherche et contrôle des cas, etc.) et sanitaires (intervention communautaire - via des visites à domicile d'infirmières et à leurs activités d'éducation hygiénico-sanitaire et la prophylaxie à travers la correction des pratiques à risque, l'amélioration des conditions des maisons, de l'hygiène personnelle et familiale, etc.).

L'expérience historique de l'Espagne et de l'Italie permet de réaffirmer la validité de ces stratégies de coopération qui affirment que pour régler un problème de santé comme une maladie infectieuse associée à la misère, la pauvreté et l'inégalité, il ne suffit pas d'agir sur les facteurs les plus immédiats, via des ressources thérapeutiques ou des interventions d'assistance. Au contraire, il s'avère nécessaire d'améliorer les conditions d'hygiène et de modifier les différents facteurs qui expliquent leur prévalence, via une intervention horizontale sur le moyen et une action prophylactique où l'éducation de la santé est fondamentale.

Université de SASSARI et Université D’ AlicAnte 